\title{
A Rare Chromosomal Disorder - 14q Interstitial Deletion Syndrome
}

\author{
Boglis Alina*, Rac Corina Dana, Moldovan Elena, Duicu Carmen, Bănescu Claudia
}

University of Medicine and Pharmacy, Tîrgu Mureș, Romania

Introduction: Interstitial deletions of the long arm of chromosome 14q (OMIM 613457) are very rare conditions. Case presentation: We present a 3-month-old male patient with dysmorphic features and congenital heart defect associated with a small interstitial deletion of chromosome 14q, identified by cytogenetic analysis as 46,XY,del(14)(q11q12). Dysmorphic features included microcephaly, broad nasal bridge, micrognathia, large and poorly folded auricular lobes and long digits. He also present rectus abdominis diastasis and umbilical hernia. The cranial computer tomography showed partial agenesis of the corpus callosum and ventriculomegaly. Conclusions: Cytogenetic analysis or molecular techniques are necessary to establish the correct diagnosis in patients with multiple congenital anomalies in association with proximal or distal interstitial $14 \mathrm{q}$ deletion.

Keywords: chromosome 14q; interstitial deletion; corpus callosum

Received: 18 July 2016 / Accepted: 05 September 2016

\section{Introduction}

Interstitial deletions of the long arm of chromosome $14 \mathrm{q}$ (OMIM 613457) are very rare conditions [1]. Most patients with this chromosomal abnormality share common clinical manifestations, such as microcephaly, micrognathia, broad nasal bridge, ear anomalies, hypotonia, developmental delay, congenital heart defects and agenesis of corpus callosum [1-22]. So far, only 27 patients with proximal interstitial deletion of chromosome 14q11-q22 have been reported, including siblings and prenatally diagnosed cases [2-12,14-22].

In this paper we present the case of a 3-month-old male patient with dysmorphic features, congenital heart defect and partial agenesis of corpus callosum with a de novo small proximal interstitial deletion of chromosome 14q, identified by cytogenetic analysis.

\section{Case presentation}

\section{Clinical Report}

In this report we present the clinical manifestations of a 3 -month-old male patient, caused by an interstitial deletion of chromosome 14q11q12.

The clinical history showed the patient to be a premature, with a gestational age of 36 weeks, coming from an unmonitored pregnancy. Birth was by Cesarean section in emergency conditions, following placental abruption. Newborn measurements indicated: birth weight 2400 grams, being considered in normal range [23], head circumference $30 \mathrm{~cm}$ and total body length $53 \mathrm{~cm}$. Apgar scores were 8 at one-minute and 9 at five-minutes, with appropriate postpartum adaptation.
Family history was negative for the present condition and there was no suspicion of consanguinity between the parents. The mother, $G_{1} P_{1}$, was without teratogenic exposures during the pregnancy.

The newborn's clinical evaluation revealed microcephaly, micrognathia, brachycephaly, broad nasal bridge, large low-set ears, high-arched palate, and other anomalies: long digits, plantar groove between hallux and $2^{\text {nd }}$ finger of the right foot, rectus abdominis diastasis and umbilical hernia.

In evolution, the newborn developed moderate generalized hypotonia, had a poor weight gain and a systolic murmur was detected, ultrasound examination revealing a wide atrial septal defect with significant shunt.

Routine neonatal transfontanellar ultrasound examinations indicated a partial agenesis of the corpus callosum associated to a moderate ventriculomegaly, confirmed also by cranial computer tomography.

The patient's evolution was unfavorable, showing a marked developmental delay associated to a poor neurologic outcome and a poor weight gain.

At the age of 3 months, the patient was diagnosed with pneumonia and developed an acute respiratory insufficiency. He was unresponsive to treatment and death occurred.

\section{Cytogenetic Analysis and Results}

The cytogenetic analysis was performed on phytohaemagglutinin M stimulated fresh peripheral blood lymphocytes, cultured for 72 hours. The karyotype was determined according to standard protocols by GTG-banding staining (G-bands by trypsin using Giemsa) and interpreted as $46, \mathrm{XY}$, del(14)(q11q12). The karyotype performed in the mother was normal, while the father refused the cytogenetic analysis. 


\section{Discussions}

Our aim was to describe a new case with a small proximal interstitial deletion involving $14 \mathrm{q} 11 \mathrm{q} 12$ bands and to review the existing literature with regard to this rare condition.

The major clinical features, reported in patients with a proximal interstitial $14 \mathrm{q} 11$ to $14 \mathrm{q} 22$ deletion are summarized in Tables I-II. Most patients have in common craniofacial features, which were less severe in our case, and developmental delay. However, these characteristics were not constantly observed and some facial features, such as long philtrum, small mouth, hypertelorism or epicanthus were also described in patients with proximal and distal $14 \mathrm{q}$ interstitial deletion [22].
Grammatico et al. presented the first case of $14 \mathrm{q} 11.2 \mathrm{q} 13$ deletion, with the phenotypic aspect of microcephaly, right plagiocephaly, bilateral cryptorchidism, and left hip subluxation [10]. A de novo 14q11.2q13 interstitial deletion was reported later by Govaerts et al., the patient presenting microcephaly, slightly protruding eyes, epicanthus, high narrow palate, short neck, arthrogryposis and unilateral renal cysts, and diabetes insipidus [9]. In their patient death occurred at the age 7 months from pneumonia. Also, other patients described in literature had frequent pneumonia or anomalies of the respiratory system $[4,9,14]$. Kamnasaran et al. reviewed the clinical features of 9 unrelated children and common features included microcephaly, hypotonia, poor growth, intellectual disability and hypoplasia of the

Table I. Clinical manifestations of patients with 14q11-q22 deletions between 1994 and 2004

\begin{tabular}{|c|c|c|c|c|c|c|c|c|c|}
\hline & $\begin{array}{c}\text { Present } \\
\text { case } \\
2013\end{array}$ & $\begin{array}{c}\text { Grammatico } \\
\text { et al. } \\
1994\end{array}$ & $\begin{array}{c}\text { Shapira } \\
\text { et al. } \\
1994(2)\end{array}$ & $\begin{array}{c}\text { Govaerts } \\
\text { et al. } \\
1996\end{array}$ & $\begin{array}{c}\text { Schuffenhauer } \\
\text { et al. } \\
1999\end{array}$ & $\begin{array}{l}\text { Ramelli } \\
\text { et al. } \\
2000\end{array}$ & $\begin{array}{c}\text { Kamnasaram } \\
\text { et al. } \\
2001 \text { (6) }\end{array}$ & $\begin{array}{l}\text { Petek et al. } \\
2003\end{array}$ & $\begin{array}{c}\text { Su PH et al. } \\
2004\end{array}$ \\
\hline $\begin{array}{l}\text { Chromosome } 14 \text { break } \\
\text { point }\end{array}$ & $\begin{array}{l}q 11 \\
q 12\end{array}$ & $\begin{array}{l}\mathrm{q} 11.2 \\
\mathrm{q} 13\end{array}$ & $\begin{array}{c}\text { q11.2 } \\
\text { q21.1/ } \\
q 12 q 22\end{array}$ & $\begin{array}{l}\mathrm{q} 11.2 \\
\mathrm{q} 13\end{array}$ & $\begin{array}{c}\mathrm{q} 11.2 \\
\mathrm{q} 13\end{array}$ & $\begin{array}{l}\mathrm{q} 11.2 \\
\mathrm{q} 13.1\end{array}$ & $\begin{array}{l}\text { q11 } \\
q 13 \\
q 22\end{array}$ & $\begin{array}{c}q 12 \\
q 13.1\end{array}$ & $\begin{array}{l}\mathrm{q} 11.2 \\
\mathrm{q} 13.1\end{array}$ \\
\hline Microcephaly & + & + & $+/+$ & + & + & + & + & + & + \\
\hline Micro(retro)-gnathia & + & & $-/+$ & & + & & + & & \\
\hline Broad nasal bridge & + & + & $-/+$ & & & + & + & & \\
\hline Ear anomalies & + & + & $-/+$ & & + & & & & \\
\hline Low-set ears & + & & $-/+$ & & + & & & & \\
\hline Congenital heart defect & + & & $-/+$ & & + & & + & & \\
\hline Agenesis corpus callosum & + & & $+/+$ & & + & & + & & + \\
\hline Hypotonia & + & & $-/+$ & & + & & + & & \\
\hline Developmental delay & & + & $+/+$ & & + & + & + & & + \\
\hline Poor growth & + & + & $+/+$ & & + & & + & & \\
\hline Epicanthus & & & & & & & & + & \\
\hline Long philtrum & & & & & & & + & + & \\
\hline Cleft lip/palate & & & & & + & & + & & \\
\hline Renal anomalies & & & & + & & & & + & \\
\hline Other anomalies & + & + & & + & & & & + & \\
\hline Pneumonia & + & & & + & & & & + & \\
\hline
\end{tabular}

+, present; - and empty cells, not-present; 0, number of cases

Table II. Clinical manifestations of patients with 14q11-q22 deletions between 2007 and 2016

\begin{tabular}{|c|c|c|c|c|c|c|c|c|c|c|c|c|}
\hline & $\begin{array}{c}\text { Our } \\
\text { case } \\
2013\end{array}$ & $\begin{array}{c}\text { Segawa } \\
\text { et al. } \\
2007\end{array}$ & $\begin{array}{l}\text { Zahir et } \\
\text { al. } 2007 \\
\text { (3) }\end{array}$ & $\begin{array}{l}\text { Papa } \\
\text { et al. } \\
2008\end{array}$ & $\begin{array}{c}\text { Mencarelli } \\
\text { et al. } \\
2009\end{array}$ & $\begin{array}{c}\text { Torgykes } \\
\text { et al. } \\
2011 \text { (2) }\end{array}$ & $\begin{array}{c}\text { Fonseca } \\
\text { et al. } \\
2011\end{array}$ & $\begin{array}{l}\text { Bravo } \\
\text { et al. } \\
2012\end{array}$ & $\begin{array}{c}\text { Gamage } \\
\text { et al. } \\
2012\end{array}$ & $\begin{array}{c}\text { Piccione } \\
\text { et al. } \\
2012\end{array}$ & $\begin{array}{c}\text { Ellaway } \\
\text { et al. } \\
2013 \text { (2) }\end{array}$ & $\begin{array}{c}\text { Gentile } \\
\text { et al. } \\
2016\end{array}$ \\
\hline $\begin{array}{l}\text { Chromosome } 14 \\
\text { break point }\end{array}$ & $\begin{array}{l}\mathrm{q} 11 \\
\mathrm{q} 12\end{array}$ & $\begin{array}{l}\mathrm{q} 13 \\
\mathrm{q} 21\end{array}$ & $\mathrm{q} 11.2$ & q12 & $q 12$ & $q 12 q 13.1 q 13$ & $\mathrm{q} 12 \mathrm{q} 13.3$ & $\begin{array}{l}\mathrm{q} 11.2 \\
\mathrm{q} 21.2\end{array}$ & $q 11.2 q 13$ & q13.1q21.1 & $\mathrm{q} 12$ & $q 13.2 q 21.1$ \\
\hline Microcephaly & + & & & + & + & $+/+$ & & + & + & & $+/+$ & + \\
\hline Micro(retro)-gnathia & + & & & & & & & + & + & & & \\
\hline Broad nasal bridge & + & & + & & & $+/+$ & + & & & & & \\
\hline Low-set ears & + & & & & & $+/+$ & + & + & + & & & \\
\hline $\begin{array}{l}\text { Congenital heart } \\
\text { defect }\end{array}$ & + & & + & & & & + & & & & & \\
\hline $\begin{array}{l}\text { Agenesis corpus } \\
\text { callosum }\end{array}$ & + & & & + & & $+/+$ & & + & + & & $+/+$ & \\
\hline Hypotonia & + & & + & & + & & + & & + & & $+/+$ & \\
\hline Developmental delay & - & & & + & + & +/+ & + & & & & +/+ & \\
\hline Poor growth & + & & & & & & + & & + & & + & + \\
\hline Epicanthus & - & & & + & + & t/t & + & & & & & \\
\hline Long philtrum & - & & + & & & $+/+$ & + & & & & & \\
\hline Cleft lip/palate & - & + & & & & & & & & & & \\
\hline Renal anomalies & - & & & & & & & & & & & \\
\hline Other anomalies & + & + & & & & $+/-$ & + & + & + & + & t/+ & + \\
\hline Pneumonia & + & & & & & $-/+$ & & + & & & & \\
\hline
\end{tabular}


corpus callosum. Other findings included congenital heart defects, underdeveloped pituitary gland, hypothyroidism, iris coloboma, hypertelorism, midface hypoplasia, bilateral cleft lip and respiratory distress [3].

Some patients with interstitial or proximal 14q deletion also associate partial or total ACC. Since ACC was also present in 12 of the 27 cases, we may suggest that the region $\mathrm{q} 11 \mathrm{q} 13$ on chromosome $14 \mathrm{q}$ includes the genes for ACC [13].

Other less frequent congenital anomalies observed in patients with $14 \mathrm{q}$ proximal interstitial deletion include congenital heart defects (CHDs) and ventriculomegaly as observed in the present case, holoprosencephaly (HPE), cleft lip/palate, renal anomalies, anophthalmia, small mouth and long philtrum, also seen in our patient. Similarly, other authors presented cases which showed these less frequent phenotypic aspects. For instance, Zahira et al. reported 3 unrelated children with a de novo interstitial deletion of chromosome 14q11.2, from which one child had a ventricular septal defect and patent ductus arteriosus [5]. Interestingly, our patient had brachycephaly, long digits, a plantar groove between the hallux and the $2^{\text {nd }}$ toe of the right foot, diastasis of the rectus abdominis and umbilical hernia, phenotypic aspects not found in previously reported cases.

\section{Conclusions}

In summary, we have reported a patient with multiple congenital anomalies in association with a de novo proximal interstitial 14q deletion. Taking into account the variation in patients' phenotype, the clinical diagnosis of $14 \mathrm{q}$ proximal or distal interstitial deletion is difficult. Cytogenetic analysis or molecular techniques are necessary to establish the correct diagnosis.

\section{Conflicts of interest}

The authors report no conflicts of interest.

\section{References}

1. Shashidhar Pai G, Lewandowski Raymond C, Borgaonkar Digamber S. Handbook of chromosomal syndromes. New Jersey, John Wiley \& Sons Inc., Hoboken, 2003. Pg215.

2. Petek E, Plecko-Startinig B, Windpassinger C, Egger H, Wagner K, Kroisel PM. Molecular characterisation of a $3.5 \mathrm{Mb}$ interstitial $14 \mathrm{q}$ deletion in a child with several phenotypic anomalies. J Med Genet. 2003;40(4):e47.

3. Kamnasaran D, O'Brien PC, Schuffenhauer S, et al. Defining the breakpoints of proximal chromosome $14 q$ rearrangements in nine patients using flow-sorted chromosomes. Am J Med Genet. 2001;102(2):173182.

4. Torgyekes E, Shanske AL, Anyane-Yeboa K, et al. The proximal chromosome $14 \mathrm{q}$ microdeletion syndrome: delineation of the phenotype using high resolution SNP oligonucleotide microarray analysis (SOMA) and review of the literature. Am J Med Genet A. 2011;155A(8):1884-
1896. DOI: 10.1002/ajmg.a.34090

5. Zahir F, Firth HV, Baross A, et al. Novel deletions of $14 \mathrm{q} 11.2$ associated with developmental delay, cognitive impairment and similar minor anomalies in three children. J Med Genet. 2007;44(9):556-561. DOI: 10.1136/jmg.2007.050823

6. Segawa Y, Itokazu N, Hirose A, Nakagawa S, Takashima S. A case of partial 14q- with facial features of holoprosencephaly and hydranencephaly. Pediatr Neurol. 2007;37(1):51-54. DOl: 10.1016/j. pediatrneurol.2007.02.010

7. Gamage TH, Godapitiya IU, Nanayakkara S, Jayasekara RW, Dissanayake $\mathrm{VH}$. A child with mosaicism for deletion (14)(q11.2q13). Indian J Hum Genet. 2012;18:130-133. doi: 10.4103/0971-6866.96684.

8. Fonseca DJ, Prada CF, Siza LM, et al. A de novo 14q12q13.3 interstitial deletion in a patient affected by a severe neurodevelopmental disorder of unknown origin. Am J Med Genet A. 2012;158A(3):689-693. doi: 10.1002/ajmg.a.35215.

9. Govaerts L, Toorman J, Blij-Philipsen MV, Smeets D. Another patient with a deletion 14q11.2q13. Ann Genet. 1996;39(4):197-200.

10. Grammatico P, De Sanctis S, Di Rosa C, Cupilari F, Del Porto G. First case of deletion 14q11.2q13: clinical phenotype. Ann Genet. 1994;37(1):3032.

11. Ramelli GP, Remonda L, Lovblad KO, Cupilari F, del Porto G. Abnormal myelination in a patient with deletion 14q11.2q13.1. Pediatr Neurol. 2000;23(2):170-172.

12. Shapira SK, Anderson KL, Orr-Urtregar A, Craigen WJ, Lupski JR, Shaffer LG. De novo proximal interstitial deletions of 14q: cytogenetic and molecular investigations. Am J Med Genet. 1994;52(1):44-50.

13. Chen CP, Lee CC, Chen LF, Chuang CY, Jan SW, Chen BF. Prenatal diagnosis of de novo proximal interstitial deletion of $14 \mathrm{q}$ associated with cebocephaly. J Med Genet. 1997;34:777-778.

14. Bravo EK, White ML, Olney AH, McAllister JL, Zhang YD. Novel Proximal 14q Deletion: Clinical and Diffusion Tensor Imaging Tractography Findings in a Patient with Lissencephaly, Agenesis of the Corpus Callosum, and Septo-Optic Dysplasia. AJNR Am J Neuroradiol. 2012;33(2):E16-18. doi: 10.3174/ajnr.A2745

15. Papa FT, Mencarelli MA, Caselli R, et al. A 3 Mb deletion in $14 q 12$ causes severe mental retardation, mild facial dysmorphisms and Rett-like features. Am J Med Genet A. 2008;146A(15):1994-1998. doi: 10.1002/ ajmg.a.32413.

16. Su PH, Chen SJ, Lee IC, et al. Interstitial deletion of chromosome $14 \mathrm{q}$ in a Taiwanese infant with microcephaly. J Formos Med Assoc. 2004;103(5):385-387.

17. Schuffenhauer S, Leifheit HJ, Lichtner P, Peters H, Murken J, Emmerich P. De novo deletion (14)(q11.2q13) including PAX9: clinical and molecular findings. J Med Genet 1999;36:233-236.

18. Mencarelli MA, Kleefstra T, Katzaki E, et al. 14q12 Microdeletion syndrome and congenital variant of Rett syndrome. Eur J Med Genet. 2009;52(23):148-152. doi: 10.1016/j.ejmg.2009.03.004.

19. Piccione M, Serra G, Consiglio V, et al. 14q13.1-21.1 deletion encompassing the HPE8 locus in an adolescent with intellectual disability and bilateral microphthalmia, but without holoprosencephaly. Am J Med Genet A. 2012;158A(6):1427-1433. doi: 10.1002/ajmg.a.35334.

20. Gentile M, De Mattia D, Pansini A, et al. $14 q 13$ distal microdeletion encompassing NKX2-1 and PAX9: Patient report and refinement of the associated phenotype. Am J Med Genet A. 2016;170(7):1884-1888. doi: 10.1002/ajmg.a.37691.

21. Ellaway CJ, Ho G, Bettella E, Knapman A, et al. $14 q 12$ microdeletions excluding FOXG1 give rise to a congenital variant Rett syndromelike phenotype. Eur J Hum Genet. 2013;21(5):522-527. doi: 10.1038/ ejhg.2012.208

22. Cingöz S, Bache I, Bjerglund L, et al. Interstitial deletion of 14q24.3-q32.2 in a male patient with plagiocephaly, BPES features, developmental delay, and congenital heart defects. Am J Med Genet A. 2011;155A(1):203206. doi: 10.1002/ajmg.a.33766.

23. Olsen IE, Groveman SA, Lawson ML, Clark RH, Zemel BS. New intrauterine growth curves based on United States data. Pediatrics. 2010;125(2):e214-224. doi: 10.1542/peds.2009-0913. 